\title{
Über welche Governance Strukturen sollte der Vorsorgestaat verfügen?
}

\author{
Sicherheit, Vorsorge, Planbarkeit: Grundbedürfnisse von Staat \\ und Gesellschaft
}

\section{Manfred Mai}

Online publiziert: 24. November 2020

(C) Der/die Autor(en) 2020

In der Erzählung „Der Bau“ von Franz Kafka ist von einer Art Dachs die Rede, der seinen Bau aus Angst vor Feinden immer weiter perfektioniert, bis er schließlich nur noch ein unerklärliches Geräusch wahrnimmt, das ihn paranoid werden lässt. Das kafkaeske Fabeltier weiß nicht einmal, ob diese Feinde überhaupt existieren. Die Erzählung ist unvollendet. Denkbar wäre, dass das Tier vor Angst darüber stirbt, dass sein scheinbar perfekter Bau eine übersehene Sicherheitslücke hat.

Diese Parabel auf den vergeblichen Versuch, absolute Sicherheit zu erreichen, passt zu den Diskursen über den Vorsorgestaat: „Sicherheit ist stets ein unerreichbares Ideal, das Bedürfnis danach nimmt nicht linear, sondern exponentiell zu den unbestreitbar gestiegenen Sicherheitsstandards zu. Bedürfnisse, Erfordernisse, Möglichkeiten und Kompetenzen der Prävention heizen sich im Laufe des Zivilisationsprozesses gegenseitig auf“ (Strasser und van den Brink 2005). Der Hinweis auf „übersehene Sicherheitslücken“ ist das Geschäftsmodell ganzer Dienstleistungsbranchen.

In der Garantie der Sicherheit vor inneren und äußeren Feinden sah Hobbes „,der Sicherheitspolitiker unter den Staatsphilosophen“ (Mack 2008) - die zentrale Aufgabe des Leviathans. Nur ein starker Staat könne seine Bürger schützen. Auch heute gehören Sicherheit und Vorsorge zu den wichtigsten Erwartungen der Bürger an den Staat. Mit fortschreitender Säkularisierung kann dieser diese Erwartungen nicht mehr ohne eine „Sicherheitslücke“ erfüllen. Seit in der amerikanischen Verfassung das christliche Weltbild durch das Recht des Einzelnen auf Streben nach Glück ersetzt wurde (Hattenhauer 1980, S. 243), ist die „Abnabelung der modernen Welt

\footnotetext{
M. Mai $(\bowtie)$

Institut für Politikwissenschaft, Universität Duisburg-Essen, Lotharstraße 65, 47057 Duisburg, Deutschland

E-Mail: manfred.mai@uni-due.de
} 
vom metaphysischen Schoß der alten Grundgewissheit" institutionalisiert. ${ }^{1}$ (Meyer 1998, S. 151) Es kann also nur darum gehen, die Risiken ,,auf das erträgliche Maß zu verringern, das der Freiheit weder durch die überzogene Einengung der Handlungsspielräume noch durch Lähmung der Angst vor der übermächtigen Unsicherheit des Lebens den Atem nimmt. Es zeigt sich [...], dass allein die Demokratie sich der Lösung dieser schwierigen Aufgabe annähern kann, dass sie zugleich aber kaum dafür gemacht ist, sie wirklich überzeugend zu lösen“" (a.a.O.: S. 157).

Der Gedanke, die Gesetzmäßigkeiten der Gesellschaft zu erkennen, um die Welt nicht nur zu interpretieren, sondern auch zu verändern, wurde von den Gründungsvätern der Soziologie aufgegriffen ${ }^{2}$. Gestützt auf Analysen über die Lage benachteiligter Gruppen sollten gezielte Interventionen in die Gesellschaft dazu dienen, ihre Lage zu verbessern. Endlich, so hofften sie, „könne Politik wissenschaftlich betrieben werden." (Maus 1967, S. 21) ${ }^{3}$ Bis heute reicht die Tradition der engen Verknüpfung zwischen Sozialforschung und Wohlfahrtsverbänden, ,sozialen und politischen Übelständen zu Leibe zu gehen.“ (Maus 1967, S. 38) Im Zentrum dieser Überlegungen stand die wachsende Armut als Folge der Industriellen Revolution. „Die im Zuge industrieller Modernisierung sich verschärfenden Spannungen und Spaltungen der Gesellschaft brachen das Vertrauen in liberale Selbstregulierung. Die zwischen ,großem Reichtum ' und ,großer Armut' aufbrechenden Gegensätze führten zur Polarisierung von ,Kapital und Arbeit‘ und zugleich zur Politisierung sozialer Konflikte." (Pankoke und Sachße 1992, S. 151) Eine Antwort auf diese Frage war die ,innere Reichsgründung“ durch soziale Reformen.

Der Gedanke, durch eine Politik der Vorsorge die Menschen vor Risiken zu schützen, war nicht nur Pate bei der Schaffung des Sozialstaats. Zur umfassenden Vorsorge gehört auch der Schutz vor Risiken durch Wissenschaft, Technik, Tierseuchen, Pandemien, Naturkatastrophen, Kriminalität und Terrorismus. Diesen anthropogenen (Kernenergie, Klimawandel) und natürlichen (Vulkanausbruch, Erdbeben) Risiken entsprechen die Politikfelder Soziales, Gesundheit, Inneres, Justiz, Technologie, Klima und Umwelt. In allen diesen Politikfeldern haben sich jeweils unterschiedliche Institutionen, Pfade und Rechtsgebiete etabliert, die aber nur Teilbereiche einer umfassenden Vorsorgepolitik abdecken.

Der Vorsorgestaat müsste über Governancestrukturen verfügen, die die Erfahrungen und Kompetenzen aus diesen fragmentierten Bereichen $\mathrm{zu}$ einer integrierten Risikopolitik zusammenführen. Aus der Sicht vieler Bürger bilden alle diese unterschiedlichen Risiken einen bedrohlichen Komplex, der einen Nährboden für fundamentalistische Ideologien bildet. Viren, Mikroplastik, Dürresommer und Kri-

\footnotetext{
1 Georg Christoph Lichtenberg hat diese Erkenntnis schon im 18. Jahrhundert auf den Punkt gebracht: „Dass in den Kirchen gepredigt wird, macht deswegen die Blitzableiter auf ihnen nicht unnötig.“.

2 „Die Aufklärer und viele ihrer Nachfolger nahmen an, zunehmendes Wissen über Gesellschaft und Natur werde zu deren zunehmender Beherrschbarkeit führen." (Giddens 1996, S. 116) Wissen und Kontrolle waren fast Synonyme. Dennoch wurde deutlich, dass die moderne Gesellschaft ,wegen und nicht trotz des Wissens" (a.a. O.) immer kontingenter und damit scheinbar unsicherer wurde.

3 Helmut Schelsky stellt fest, dass ,erst mit der Geburt der Soziologie bei Saint-Simon und Comte [...] ,der Plan“ zum beanspruchten Privileg einer Wissenschaft [wurde], die zur Herrschaft drängte." So werde die Gesellschaft in aktive Planer und passiv Beplante gespalten. Dennoch lässt Schelsky keinen Zweifel daran, dass ,planendes, vorausschauendes Handeln zur Natur des Menschen“ gehört. (Schelsky 1976, S. 100).
} 
minalfälle werden dann zu einem finsteren Szenario zusammengerührt, dass nur ein radikaler Systemwechsel als Ausweg erscheint. Der gemeinsame Nenner ist ein wahrgenommener Kontrollverslust, der der Politik angelastet wird.

\section{Der Sozialstaat als Antwort auf soziale Risiken}

Von allen vorsorgepolitisch relevanten Politikbereichen ragt der Bereich „Soziales“ hervor. Die Anzahl der für ",Soziales“ tätigen Institutionen, sein politische Konfliktpotenzial und die dafür aufgewendeten Kosten überragen deutlich die der anderen Bereiche. Das in den 1880er-Jahren von Bismarck ${ }^{4}$ geschaffene Modell der sozialen Sicherung „,sah einen dauerhaften, institutionalisierten und durch Rechtsansprüche abgesicherten effektiveren Schutz gegen die Risiken einer arbeitsteiligen Industriegesellschaft“ (Schmidt 1988, S. 22) vor. Ernst Forsthoff sprach von einer „existenziellen Notwendigkeit der Daseinsbewältigung in der industriellen Moderne“, die ,den Staat notwendig zum Sozialstaat macht" (Meinel 2019, S. 102). Er und andere konservative Autoren warnten auch vor den negativen Konsequenzen des Sozialstaats. ${ }^{5}$ Helmut Schelsky kritisierte z.B., dass in der Verbreitung des Bewusstseins, ,dass fast alle zu den Hilfsbedürftigen gehören“, eine neue Legitimitätsquelle für den Staat entsteht (Schelsky 1976, S. 100). Fürsprecher des Sozialstaats sehen dagegen in der "Sicherung der Loyalität der Staatsbürger" und in der Einhegung von Interessengegensätzen seine wichtigsten Funktionen (Schmidt 1988, S. 187, Nullmeier 2013). Der Traum der Sozialdemokratie ist der Albtraum der Liberalen: Ein Staat, der kollektive Sicherheit höher schätzt als individuelle Freiheit. Zwischen diesen Polen muss jede Architektur kollektiver Vorsorge angelegt sein, wenn sie demokratisch legitimiert werden soll und sich auf die politischen Kräfte in der Gesellschaft stützt.

Sozialleistungen, Präventionsmaßnahmen und Hilfsprogramme für Bedürftige müssen finanziert werden. So wie die Wirtschaft von der Stabilisierungsleistung des Sozialstaats abhängt, so sind Sozialleistungen abhängig vom Wirtschaftswachstum. (Kaufmann 1997, S. 83). Der Sozialstaat kommt an seine Grenzen. Die seit den 1970er-Jahren geführten Debatten über seine Zukunft haben sich seitdem verstärkt (Heinze et al. 1987; Boeßenecker et al. 2000). Gründe dafür sind:

- Sozialer und demografischer Wandel,

- Strukturwandel in Industrie,

- Umbrüche in der Arbeitswelt und

- Wertewandel.

\footnotetext{
${ }^{4}$ Als Idealtyp eines ostelbischen Junkers war Bismarck die Hassfigur der Sozialisten und Sozialdemokraten. Ausgerechnet er hat dem Sozialstaat durch seine Sozialgesetze den Boden geebnet (Grimm 1987, S. 151; Schmidt 1988, S. 21). Zeigt sich Hegels Weltgeist nicht gerade darin, dass er hinter dem Rücken der Akteure die Menschheit zu immer höherer Vernunft führt? „Bei aller Gegnerschaft gegen die Innenpolitik des reaktionären Sozialistenfressers erkannten Marx und Engels in Bismarck ,einen Agenten des Hegelschen Weltgeistes““. (Nick Brauns in: „Junge Welt“ vom 25. August 2020: „Lasst Bismarck auf dem Sockel. Plädoyer für einen dialektischen Umgang mit der Geschichte.“ (Quelle: https://www.jungewelt.de/ artikel/384947.lasst-bismarck-auf-dem-sockel.html (Abgerufen am 25.08.2020)).
}

${ }^{5}$ Zur politikwissenschaftlichen Sozialstaatsdiskussion: Trampusch (2009, S. 200). 


\section{Prävention als politisches Programm}

Das Verhältnis zwischen Staat, Markt und Dritter Sektor wird sich wegen dieser Entwicklungen weiterhin ändern. In einer Arbeitswelt, die zunehmend aus kreativen Netzwerken und Projekten besteht (Reckwitz 2017, S. 107), hat das klassische Verständnis von Sozialstaat fast keine Bedeutung mehr. Statt das Geld für Sozialprogramme auszugeben, sollte es nach liberaler Ansicht besser in Bildung, Innovationen, Bürgergeld und Startups investiert werden. Aus sozialdemokratischer Sicht wird dagegen mit Blick auf Skandinavien der Sozialstaat mit dem Leitbild der Prävention weitergedacht: Durch Investitionen in Vorsorge sollen spätere Folgekosten erspart werden. Ob und in welcher Form sich später Einsparungen - die Präventionsrendite - zeigen, ist schwierig nachzuweisen.

Dieser präventive Ansatz wurde von der rot-grünen Landesregierung NRW 2010 Teil des Regierungsprogramms, was die damalige Opposition 2013 zu einer Kleinen Anfrage an die Landesregierung veranlasste. Ministerpräsidentin Kraft, so die Kritik, ,hat mit den genannten Maßnahmen, die nur in einem Bruchteil der nordrhein-westfälischen Kommunen durchgeführt werden, stets eine exzessive Ausgabenpolitik [...] begründet. "6 Die Landesregierung betont in ihrer Antwort,

dass sich besonders Bildungsinvestitionen in den ersten Lebensjahren über den späteren Bildungserfolg auszahlen - gerade auch bei Kindern aus sozial schwächerem Umfeld. Davon hat nicht nur der Einzelne etwas: Geringere Transferleistungen, höhere Sozialversicherungsbeiträge und zusätzliche Steuerzahlungen im späteren Erwerbsleben führen dazu, dass der Staat die im Vorschulbereich investierten Gelder fast dreifach zurückbekommt. (a. a. O.)

Der plausibel klingende Ansatz - was wir an Vorsorge investieren, ersparen wir an Nachsorge - bedeutet zunächst einen weiteren Ausbau bestehender Strukturen. Ebendies ist politisch umstritten, weil die späteren Einsparungen nicht eindeutig einer bestimmten Politik zugerechnet werden können. Es gibt aber noch einen anderen Grund, diesen Präventionsansatz zu kritisieren: Um gezielte Interventionen zu rechtfertigen, müssen Individualdaten über den Erfolg in den einzelnen Gliedern der Präventionskette erhoben werden. „Präventive Logik ist expansiv: Wer vorbeugen will, weiß nie genug“ (Prantl 2008, S. 63).

Risikobereiche außerhalb sozialpolitischer Fragen werden von diesem Ansatz kaum berührt, obwohl auch in der technischen Sicherheit ähnlich argumentiert wird: Was an Sicherheit mit eingebaut wird, senkt die potenzielle Schadenshöhe und damit die Versicherungsprämie. „Im Unterschied zu ,modernen“ Gesellschaften, in denen die ungleiche Verteilung von Wohlstand und Reichtum als zentrales Konfliktfeld des Politischen identifiziert werden könne, kennzeichne ,spätmoderne ' Gesellschaften die Expansion kollektiver Risiken und Gefahren, welche wiederum als ,Nebenfolgen “ aus der technischen Entwicklung der Industriemoderne resultierten. ,Not lässt sich

\footnotetext{
6 Antwort der Landesregierung auf die Kleine Anfrage vom 25. Juli 2013 der FDP (Drucksache 16/3693): „Ergebnisse der angeblich vorsorgenden Sozialpolitik in Nordrhein-Westfalen - Welche aus der neuen Philosophie konkret resultierenden Minderausgaben bilden sich bislang im Landeshaushalt ab?" Zum Erfolg des Präventionsansatzes siehe die Studie der Bertelsmann-Stiftung. (Lohse et al. 2017).
} 
ausgrenzen, die Gefahren des Atomzeitalters nicht mehr"“ (Wehner mit Bezug auf Ulrich Becks Konzept der „Risikogesellschaft“ 2012, S. 581).

In China ist der Ansatz vorsorgender Politik breiter gefasst und eher ein gesamtgesellschaftliches Steuerungsinstrument. Er basiert auf einem Punktesystem, das individuelles Verhalten belohnt oder sanktioniert ${ }^{7}$. Bei Fehlverhalten wird der Betreffende z. B. von bestimmten Vergünstigungen ausgeschlossen und auf einer Liste veröffentlicht. Ermöglicht wird das durch konsequenten Einsatz digitaler Technologien. Der Präsident Chinas sieht dies als einen Baustein auf dem Weg zum Ziel, die Armut in China abzuschaffen.

Auch in den westlichen Ländern werden von Sicherheitsbehörden Technologien (z.B. Gesichtserkennungssoftware, Data Mining) angewandt, um Verbrechen voraussagen und potenzielle Täter gezielt ansprechen zu können (Predictive Policing) ${ }^{8}$. Konkret geht es um Personen aus einem schwierigen Umfeld (Drogenmilieu, Gangs), die man durch Ansprache daran hindern will, so weiterzumachen wie bisher. Was in Deutschland bei Datenschützern und Menschenrechtsaktivisten umstritten ist, wird in China und anderen Staaten weiter ausgebaut, offenbar mit Billigung der Bevölkerung. Besonders in der Corona-Krise scheinen sich die zentralistischen Sicherheitsarchitekturen Chinas, Singapurs und Südkoreas zu bewähren, während die EU wie ein Flickenteppich unterschiedlicher Vorschriften aussieht.

Es zeigt sich, dass nicht nur Strukturen und Kompetenzen bei der Bekämpfung von Pandemien eine Rolle spielen, sondern auch die politische Kultur: Was sind die Bürger bereit, als Einschränkungen zu akzeptieren und wie groß ist ihr Vertrauen in den Staat? So stellen z. B. die Niederlande mit ihrer individualistischen Kultur ihren bisherigen Ansatz beim Kampf gegen Corona angesichts steigender Fälle in Frage:

Völkerpsychologie steht hoch im Kurs. Der niederländische Geriater Rudi Westendorp zog in De Volkskrant einen Vergleich mit Dänemark [...]. Dort sei das Virus erfolgreicher eingedämmt worden, weil sich der Einzelne intensiver als Teil einer Gruppe verstehe. „Du bist Teil der Gesellschaft, also muss die Gesellschaft für dich sorgen. Aber viel wichtiger ist, dass du dafür sorgst, dass die Gesellschaft gut funktioniert. "In den Niederlanden fehle dieses „kollektive Bewusstsein“. Noch dazu würden einmal gefasste Beschlüsse nicht allgemein akzeptiert. (Kirchner 2020)

\footnotetext{
7 „Die politische Führung in Peking will mit dem Sozialkreditsystem die Menschen zu moralisch einwandfreien Bürgern erziehen. Es soll dazu dienen, die Ordnung des Marktes und die Ordnung in der Gesellschaft zu verbessern. Im Sinne des Leitbildes von Chinas Staatspräsident Xi Jinping: der ,harmonischen Gesellschaft “". https://www.deutschlandfunkkultur.de/chinas-sozialkredit-system-auf-dem-wegin-die-it-diktatur.979.de.html?dram:article_id=3951 (abgerufen am 15.10.2020).

8 Eine Predictive Analytics Software versucht, „Verhaltensmuster aus Echtzeitdaten und aus der Vergangenheit bekannten Daten zu extrahieren, um damit für die Zukunft Vorhersagen zu machen. Voraussichtliche Verbrecher werden insofern bestraft, dass sie auf Listen mit potenziellen Straftätern landen." (https:// netzpolitik.org/2018/pre-crime-dokumentation-ueber-predictive-policing-in-der-arte-mediathek/ abgerufen am 15.10.2020).
} 


\section{3 „Governance by Fear“" - Lehren aus der Corona-Krise}

Es gab in den letzten Jahren mehrere Ereignisse, die die Politik zu schnellen Reaktionen zwang: „9/11“, Finanzkrise 2009/10, Fukushima 2011 und jetzt die CoronaPandemie. Dazwischen liegen ,normale Katastrophen“ wie Erdbeben, Terror, Kriege, Hungersnöte, Tsunamis, Hochwasser, Taifune, Dürren. Für die Politik sind auch normale Katastrophen eine Herausforderung, die sich aber in erprobten Routinen bewältigen lassen: Es gibt klare Zuständigkeiten, Meldeketten und Kompetenzen. Versicherungen können diese Ereignisse versicherungsmathematisch modellieren und auf dieser Basis Prämien berechnen. Singuläre Katastrophen entziehen sich dieser Berechenbarkeit und erfordern politische Entscheidungen, die bewährte Routinen in Frage stellen und Verfassungsprinzipien wie Gewaltenteilung, Föderalismus und Grundrechte betreffen. Die „Entscheidungszumutung“ (Korte 2020) besteht darin, zeitnah die richtigen Entscheidungen zu treffen und umzusetzen. Krisen erlauben kein Zögern und der Normalfall demokratischer Kultur, das Suchen nach Lösungen, das Einbinden möglichst vieler Interessen, das Organisieren von Mehrheiten und das öffentliche Werben für eine bestimmte Lösung, kann Krisen noch verschärfen.

Problematisch dabei ist die Legitimität dieser Entscheidungen. Reicht die „Herrschaft kraft Wissen“ der Experten oder muss gerade unter Stressbedingungen die Demokratie Vorrang haben: Legitimation durch demokratische Verfahren. Diese Extremsituationen zeigen das Dilemma zwischen Sicherheit und Freiheit. In jeder Krise stellt sich die Frage, wieviel Freiheit wir aufzugeben bereit sind. Dabei kann es nur darum gehen, diesen temporären Krisenmodus dann zu beenden, wenn die Krise vorbei ist. Wer bestimmt das und welche Rollenverteilung besteht dabei zwischen Parlament, Exekutive und Experten? Das Bundesverfassungsgericht stellte dazu fest:

Das Grundgesetz spricht dem Parlament nicht einen allumfassenden Vorrang bei grundlegenden Entscheidungen zu. Es setzt durch die gewaltenteilende Kompetenzzuordnung seinen Befugnissen Grenzen. Weitreichende - gerade auch politische - Entscheidungen gibt es der Kompetenz anderer oberster Staatsorgane anheim, wie zum Beispiel die Bestimmung der Richtlinien der Politik durch den Bundeskanzler (Art. 65 Satz 1 GG), die Auflösung des Bundestages (Art. 68 GG), die Erklärung des Gesetzgebungsnotstands (Art. 81 GG) oder wichtige außenpolitische Entscheidungen, wie etwa über die Aufnahme oder den Abbruch diplomatischer Beziehungen.

Dem Bundestag, der solche Entscheidungen mißbilligt, verbleiben seine Kontrollbefugnisse [...].

(BVerfG 1978, Beschluss vom 08.08.1978-2 BvL 8/77)

Im Zusammenhang mit den Risiken der Kernenergie hatte das Bundesverfassungsgericht festgestellt, dass der Bürger ein Restrisiko als „,sozialadäquate Last“ zu tragen habe,

wenn es nach dem Stand der Wissenschaft und Technik praktisch ausgeschlossen werden könne. Es obliege der Legislative und der Exekutive, die zu treffenden Vorsorgemaßnahmen zu ergreifen sowie die Grenzziehung zwischen hinnehmbaren und nicht hinnehmbaren Risiken durch wertende Entscheidung anhand der Zumutbarkeit festzulegen. (a.a.O.) 
Der Staat kann und muss die Bürger also nicht gegen alle denkbaren Szenarien absichern. Die politisch auszuhandelnde Frage ist, wie hoch dieses Restrisiko sein soll und wer es bestimmt. Im Atomrecht geht es um technische Details von Sicherheitsarchitekturen und Komponenten. Zentraler Bezugspunkt ist der unbestimmte Rechtsbegriff ,Stand der Technik“:

Bei der Formel vom Stand der Technik gestaltet sich die Feststellung und Beurteilung der maßgeblichen Tatsachen für Behörden und Gerichte allerdings schwieriger. Sie müssen in die Meinungsstreitigkeiten der Techniker eintreten, um zu ermitteln, was technisch notwendig, geeignet, angemessen und vermeidbar ist. (a.a. O.)

Auch in der Corona-Krise geht es um Meinungsstreitigkeiten der Experten. Die „Erosion der Expertenautorität“" (Wehner 2012, S. 591) und ihre Politisierung begann mit der Debatte über die Atomenergie in den 1970er-Jahren: „Die Fraktionierung der Experten ist inzwischen schon fast zu einer Selbstverständlichkeit geworden. [...] Wissenschaftlicher Dissens wird in den politischen Raum verlegt und unter Regeln politischer Debatte geführt“ (Weingart 1979, S. 15). Bei der Bekämpfung der Corona-Pandemie gibt es ebenfalls eine Vielfalt unterschiedlicher Expertenmeinungen. So kann sich die Politik sowohl für Lockerungen als auch für Verschärfungen jeweils auf unterschiedliche Experten berufen. Der Dissens der Experten macht sie, wie bereits die Debatte um die Kernenergie gezeigt hat, für politische Ziele instrumentalisierbar:

Er (Hendrik Streeck) ist halt nicht der Regierungsvirologe, zu wenig alarmistisch, zu kritisch gegenüber \#governance by \#fear. Für diese Politik eignen sich andere besser, die auf Verbote setzen und Infektionszahlen als hinreichende Legitimation für die Suspendierung von \#Grundrechten halten.

(Wolfgang Merkel, Tweet vom 10.10.2020)

Auf Streeck beruft sich eine Politik der ,verantwortungsvollen Normalität“: Sie akzeptiert die Gefährlichkeit des Virus und erwartet in absehbarer Zeit keine Wundermittel. Sie will daher die Menschen darauf einstellen, dass sie mit dem Virus leben müssen. Es geht auch anders:

Governance by (rational) fear. Star-Virologist Drosten, chancellor Merkel, Jens Spahn are competing in worst case scenarios for the fall. Their main indicator: Infection rates. Many experts criticize the onedimensional infection rates scenario. Nevertheless, infections rates are suitable to create fear and compliance. There is a political logic behind governance by (scientifically created) fear: politicians who dare not to govern by worst case scenarios, will be politically killed in elections if a worst case act. occur. That leads to a pol. preference of security over freedom. Esp. green and left politicians opt for fear \& security. (Wolfgang Merkel, Tweet vom 10.10.2020) 


\section{Neue Governance Strukturen?}

Das Kalkar-Urteil warnte damals vor einer Überforderung der Politik:

Vom Gesetzgeber im Hinblick auf seine Schutzpflicht eine Regelung zu fordern, die mit absoluter Sicherheit Grundrechtsgefährdungen ausschließt, die aus der Zulassung technischer Anlagen und ihrem Betrieb möglicherweise entstehen können, hieße die Grenzen menschlichen Erkenntnisvermögens verkennen und würde weithin jede staatliche Zulassung der Nutzung von Technik verbannen. Für die Gestaltung der Sozialordnung muss es insoweit bei Abschätzungen anhand praktischer Vernunft bewenden. Ungewissheiten jenseits dieser Schwelle praktischer Vernunft sind unentrinnbar und insofern als sozialadäquate Lasten von allen Bürgern zu tragen. (BVerfG 1978, Beschluss vom 08.08.1978-2 BvL 8/77)

Was gilt heute als sozialadäquat? An den Reaktionen der Bürger auf die Maßnahmen zur Corona-Pandemie zeigt sich, dass es im Großen und Ganzen Verständnis für die beschlossenen Maßnahmen gibt. Es gibt aber nicht nur an den Rändern des politischen Spektrums Stimmen, die bereits das als Eingriff in Grundrechte sehen. In der Kritik an den Maßnahmen gegen die Corona-Pandemie treffen sich Traditionsbestände der Konservativen Revolution mit konstruktivistischen Theorien der Biopolitik. ${ }^{9}$

Grundlagen für eine angemessene Governance im Umgang mit der Pandemie sollten neben verfassungsrechtlichen und demokratietheoretischen Prinzipien auch konkrete Erfahrungen sein, die aus anderen Ländern vorliegen. Als erfolgversprechend gelten z.B. die Maßnahmen in Taiwan und Südkorea, die die Potenziale digitaler Technologien voll ausschöpfen (Digital Tracing): Die Polizei ,,wertet das GPS-Signal des Smartphones des Infizierten aus. Ist das unergiebig, schickt die Polizei ein schnelles Einsatzteam aus, das die Wohnung des Infizierten aufsucht und seine Kreditkartenzahlungen sowie öffentliche Überwachungskameras auswertet." ${ }^{\text {"10 }}$ Damit werden $80 \%$ in Südkorea der Infektionen rasch aufgeklärt.

Ein solcher Weg ist nur in Staaten denkbar, wo eine andere politische Kultur herrscht. Selbst wenn bei uns $80 \%$ der Infektionen rasch aufgeklärt würden, hätten die damit verbundenen Maßnahmen in Deutschland keine Chance. Empfehlungen für eine Governancestruktur in einem idealen Vorsorgestaat werden sich erst dann begründen lassen, wenn eine Best Practice im Umgang mit der Corona-Pandemie vorliegt. Schweden, Großbritannien oder Asien zeigen, dass jeder Erfolg seinen Preis hat und nicht jede Strategie eins-zu-eins in andere Staaten übertragen werden kann.

\footnotetext{
9 „Bei ,Biopolitik‘ denken die einen an eine rationale und demokratische Gestaltung der Lebensverhältnisse, während andere sie mit Praktiken der Aussonderung, mit Krankenmord, Eugenik und Rassismus in Verbindung bringen. Der Begriff taucht bei Vertretern der Alten Rechten ebenso auf wie in neueren linksradikalen Texten, ihn verwenden Kritiker des biotechnologischen Fortschritts, aber auch dessen Befürworter, erklärte Rassisten wie bekennende Marxisten." (Lemke 2008, S. 72).

10 https://www.youtube.com/watch?v=05jwyl8b-_c Zugegriffen: 14.10.2020.

https://www.nzz.ch/amp/technologie/contact-tracing-das-erfolgsrezept-von-suedkorea-und-taiwan-ld. 1581409? mktcid=smch\&mktcval=twpost_2020-10-14\&_twitter_impression=true Zugegriffen: 14.10 . 2010.
} 
Eine Regierung im permanenten Krisenmodus ist das Ende der Demokratie. Wie es aussieht, werden wir mit Corona leben müssen, so wie mit allen anderen Risiken, die nur kurzfristig von der Agenda verschwunden sind.

Problematisch ist vor allem die Legitimität der beschlossenen Maßnahmen, die auf Beschlüssen beruhen, die die Bundeskanzlerin mit den Ministerpräsidenten trifft. Seit dem Lockdown März 2020 ,,sind sieben Monate vergangen und man hat noch immer kein hinreichendes rechtliches Instrumentarium für epidemische Notlagen von nationalem Ausmaß geschaffen. [...] Wir brauchten ein Maßnahmengesetz für den epidemischen Notstand nationalen Ausmaßes. Den regionalen Besonderheiten könnte man Rechnung tragen durch Öffnungsklauseln für die Exekutive in den jeweiligen Ländern.“ (Papier 2020).

Das Parlament geht mit seiner berechtigten Forderung nach mehr Beteiligung in der aktuellen Krise ein Risiko ein. Sollte sich zeigen, dass Maßnahmen zwar verfassungsrechtlich sauber, aber zu ineffektiv sind, wird man es auch für das Versagen im Kampf gegen die Pandemie verantwortlich machen. Wer die Backen aufbläst, muss auch Pfeifen: Im Zweifel wird die Output-Legitimation - eine erfolgreiche Strategie gegen die Pandemie - höher geschätzt werden als eine idealtypische Input-Legitimation. Schon bei der Begründung der Notstandsgesetze hatte der Abgeordnete Hermann Busse (FDP) davor gewarnt, ,dass man die Stunde der Not zur Stunde der Exekutive macht“ und zugleich bezweifelt, ob der „große Apparat Bundestag“ in dieser Stunde der Not seine Funktionsfähigkeit erhalten kann. (zitiert in: Hofmann und Maus 1967, S. 16) Damals gab es in den Parlamenten nur drei Parteien. Heute sind es mehr als doppelt so viele mit unterschiedlichen Koalitionsoptionen auf allen Politikebenen. Das erschwert langfristige Strategien. Die Feststellung von Joachim Raschke gilt nicht nur für Parteien, sondern auch für das Parlament: „Mittel- und längerfristig können Parteien ohne Beiträge zur Problemlösung nicht erfolgreich sein. Populistische Strategien vermögen solche Defizite nur zeitlich begrenzt zu überspielen. Tatsächliche und wahrgenommene Problemlösungskompetenzen sind für Wahlen, Koalitionsbildungen, Regierungspolitik strategische wichtige Steuergrößen." (Raschke 2002, S. 222).

Die aktuelle Feststellung des ehemaligen Verfassungsrichters Hans-Jürgen Papier (2020) bleibt ebenso im Prinzipiellen wie das damalige Kalkar-Urteil zur Kernenergie: „Das verfassungsrechtlich legitime Anliegen, die Gesundheit und das Leben der Bevölkerung zu schützen, berechtigt nicht zu Freiheitseinschränkungen jedweder Art." (a.a. O.) Politiker wollen aber nicht wissen, wo die Zäune sind, sondern die Löcher. Es bleibt unklar, mit welchen konkreten Institutionen und Verfahren dieser Prozess der Abwägung zwischen Freiheitseinschränkungen und Schutz der Gesundheit getroffen werden soll. Das Verfassungsrecht kann nur Prinzipien der Rechtsstaatlichkeit auf eine konkrete Situation anwenden und feststellen, was nicht den Grundsätzen der Verfassung entspricht. Das „Wie“ ist die ureigene Aufgabe der Exekutive, die das „Was“ der Legislative und das „so nicht“ der Judikative umsetzt. Eine Governance der Vorsorge muss Antworten auf folgende Fragen finden: 
- Was ist mit den gegebenen Mehrheiten und Strukturen hier und jetzt machbar und finanzierbar?

- Was ist politisch verantwortbar?

- Was hält (Verfassungs)klagen stand?

Die Verwaltungsgerichte haben (Stand Oktober 2020) bereits mehr als 60 Verordnungen der Exekutive, die die Pandemie eindämmen sollen, gekippt. Das Recht kennt zwar viele „Zäune“, es kann aber nicht sagen, wie eine Governancestruktur konkret aussehen sollte, um die Bedingungen für eine erfolgreiche Bekämpfung der Pandemie zu verwirklichen und wie man aus abstrakten Grundsätzen konkrete Institutionen schafft: Ein ständiger Ausschuss des Bundestages z. B. oder ein „Wohlfahrtsausschuss“" mit umfassenden Kompetenzen auf Zeit? Das eröffnet Spielräume für Experimente, bei denen man Erfahrungen anderer Länder mit den Grundsätzen unserer Verfassung kombiniert. Wer heilt, hat Recht. Die Urteile über CoronaMaßnahmen (z. B. das Beherbergungsverbot) zeigen, dass die Einschränkungen der Freiheitsrechte sehr gut begründet werden müssen. Nebenbei zeigen diese Urteile auch, dass die Judikative die Defizite des Parlaments kompensiert.

Die „Spiegelaffäre“ und die Auseinandersetzungen mit den „Notstandsgesetzen“ (Hofmann und Maus 1967; Oberreuter 2018) waren in den 1960er-Jahren prägende Ereignisse für die politische Kultur in der Bundesrepublik die zeigten, dass die Bürger Einschränkungen demokratischer Rechte nicht hinnehmen. In den 1980er-Jahren hat es Warnungen vor dem „Atomstaat“ gegeben, der ebenfalls Einschränkungen von Rechten vorsah, um die Sicherheit kerntechnischer Anlagen zu rechtfertigen, (Meyer-Tasch 1984) Die daran anschließenden Diskurse über eine Risikopolitik haben die demokratietheoretische Dimension immer mitreflektiert. (Conrad 1983; Bechmann 1993; Wehner 2012) ${ }^{11}$ In diesen Kontext gehört auch die jetzige Diskussion über das Corona-Regime. Die konkrete Bedrohung ist eine andere, aber die Demokratie darf auch hier nicht zur Disposition stehen. Jede Governance-Struktur muss sich nicht nur an ihrer Effizienz, sondern auch an ihrer Demokratieverträglichkeit messen lassen. Das bedeutet: Wir müssen mit Risiken zu leben lernen, auch wenn es kein wirklicher Trost ist, dass der Verlust der Gewissheit ein Kennzeichen der Moderne ist, die ,darum zur Epoche der unentrinnbaren, generalisierbaren Ungewissheit geworden (ist)“ (Meyer 1998, S. 151).

Wir werden also wie das kafkaeske Fabeltier mit der Vorstellung leben müssen, dass unser „Bau“ übersehene Sicherheitslücken hat. Anders als diese Wühlmaus sollten wir wissen, dass die Geräusche, die wir zu hören glauben, zu den Bedingungen der Welt gehört, die wir selbst geschaffen haben.

Funding Open Access funding enabled and organized by Projekt DEAL.

Open Access Dieser Artikel wird unter der Creative Commons Namensnennung 4.0 International Lizenz veröffentlicht, welche die Nutzung, Vervielfältigung, Bearbeitung, Verbreitung und Wiedergabe in jeglichem Medium und Format erlaubt, sofern Sie den/die ursprünglichen Autor(en) und die Quelle ordnungsgemäß nennen, einen Link zur Creative Commons Lizenz beifügen und angeben, ob Änderungen vorgenommen wurden.

11 Die damaligen Kritiker kamen aus dem linken Spektrum und waren Vorläufer der ,68er“, die die politische Kultur nachhaltig prägten. Heute sind die Kritiker eher aus dem rechten Spektrum. 
Die in diesem Artikel enthaltenen Bilder und sonstiges Drittmaterial unterliegen ebenfalls der genannten Creative Commons Lizenz, sofern sich aus der Abbildungslegende nichts anderes ergibt. Sofern das betreffende Material nicht unter der genannten Creative Commons Lizenz steht und die betreffende Handlung nicht nach gesetzlichen Vorschriften erlaubt ist, ist für die oben aufgeführten Weiterverwendungen des Materials die Einwilligung des jeweiligen Rechteinhabers einzuholen.

Weitere Details zur Lizenz entnehmen Sie bitte der Lizenzinformation auf http://creativecommons.org/ licenses/by/4.0/deed.de.

\section{Literatur}

Bechmann, Gotthard (Hrsg.). 1993. Risiko und Gesellschaft. Opladen: Westdeutscher Verlag

Boeßenecker, Karl-Heinz, Achim Trube, und Norbert Wohlfahrt (Hrsg.). 2000. Privatisierung im Sozialsektor. Rahmenbedingungen, Verlaufsformen und Probleme der Ausgliederung sozialer Dienste. Münster: Votum.

BVerfG, Beschluss vom 08.08.1978-2 BvL 8/77 (https://openjur.de/u/166332.html - Zugegriffen: 11. Okt. 2020)

Conrad, Jobst. 1983. Gesellschaft, Technik und Risikopolitik. Berlin, Heidelberg: Springer.

Giddens, Anthony. 1996. Leben in einer posttraditionalen Gesellschaft. In Reflexive Modernisierung. Eine Kontroverse, Hrsg. U. Beck, A. Giddens, und S. Lash, 113-194. Frankfurt/Main: Suhrkamp.

Grimm, Dieter. 1987. Die sozialgeschichtliche und verfassungsrechtliche Entwicklung zum Sozialstaat. In Recht und Staat der bürgerlichen Gesellschaft, Hrsg. D. Grimm, 138-161. Frankfurt/Main: Suhrkamp.

Hattenhauer, Hans. 1980. Die geistesgeschichtlichen Grundlagen des deutschen Rechts. Zwischen Hierarchie und Demokratie, 2. Aufl., Heidelberg, Karlsruhe: UTB.

Heinze, Rolf G., Bodo Hombach, und Henning Scherf (Hrsg.). 1987. Sozialstaat 2000. Auf dem Weg zu neuen Grundlagen der sozialen Sicherung. Bonn: NG.

Hofmann, Werner, und Heinz Maus. 1967. Notstandsordnung und Gesellschaft in der Bunderepublik Deutschland. Reinbek: rororo.

Kaufmann, Franz-Xaver. 1997. Herausforderungen des Sozialstaats. Frankfurt/Main: Suhrkamp.

Kirchner, Thomas. 2020. Gutgelaunt scheitern. https://www.sueddeutsche.de/politik/niederlande-pandemiefalle-1.5082160. Zugegriffen: 19. Okt. 2020.

Korte, Karl Rudolf. 2020. Politische Entscheidungszumutungen: Deutsche Einheit 1989/90 und CoronaPolitik 2020. Zeitschrift für Politikwissenschaft. https://doi.org/10.1007/s41358-020-00218-3.

Lemke, Thomas. 2008. Eine Analytik der Biopolitik. Überlegungen zu Geschichte und Gegenwart eines umstrittenen Begriffs. Behemoth. A Journal on Civilisation 1:72-89.

Lohse, Katharina, Thomas Meysen, Stephanie Götte, Nerea González Méndez de Vigo, und David Seltmann. 2017. Akteursübergreifende Prävention. Verknüpfte Planung, Umsetzung und Finanzierung von Präventionsketten. Möglichkeiten der Etablierung eines kommunalen „Präventionstopfs“. Gütersloh: Bertelsmann.

Mack, Susanne. 2008im. Besprechung von Quentin Skinner: Freiheit und Pflicht. Thomas Hobbes' politische Theorie. 2008 im DLF. https:/www.deutschlandfunkkultur.de/die-grundlagen-der-freiheit.950. de.html?dram:article_id=136521. Zugegriffen: 20. Okt. 2020.

Maus, Heinz. 1967. Zur Vorgeschichte der empirischen Sozialforschung. In Geschichte und Grundprobleme Handbuch der empirischen Sozialforschung, Bd. 1, Hrsg. René König, 21-56.

Meinel, Florian. 2019. Staat und Staatlichkeit in der industriellen Moderne. Variationen eines Problems bei Ernst Forsthoff. In Von Staat zu Staatlichkeit. Beiträge zu einer multidisziplinären Staatlichkeitswissenschaft, Hrsg. Gunnar F. Schuppert, 101-116. Baden-Baden: Nomos.

Merkel, Wolfgang. „Ich nenne das: Regieren durch Angst“ - Wie demokratisch ist die Corona-Politik noch? Wolfgang Merkel kritisiert einen Alarmismus der Regierenden und die Zurückhaltung des Parlaments. Interview: Lenz Jacobsen. https://www.zeit.de/amp/politik/deutschland/2020-10/ corona-politik-demokratie-angela-merkel-regierung-pandemie-wolfang-merkel. Zugegriffen: 20. Okt. 2010.

Meyer, Thomas. 1998. Der Verlust der endgültigen Wahrheit. In Wieviel Risiko braucht die Gesellschaft?, Hrsg. der Gesamtverband, Versicherungswirtschaft, 146-169. Berlin: Gesamtverband der Deutschen Versicherungswirtschaft e.V. 
Meyer-Tasch, Peter C. 1984. Der Weg vom Rechtsstaat zum Atomstaat wird gepflastert. In Recht und Technik im Spannungsfeld der Kernenergiekontroverse, Hrsg. Alexander Roßnagel, 67-87. Opladen: Westdeutscher Verlag.

Nullmeier, Frank. 2013. Sozialstaat. In Handwörterbuch des politischen Systems der Bundesrepublik Deutschland, Bd. 7, Hrsg. Uwe Andersen, Wichard Woyke. Heidelberg: Springer VS.

Oberreuter, Heinrich. 2018. Notstandsverfassung 1968: Die Kompetenz des Parlaments und die Blamage seiner Gegner. ZParl 49(4):855-870.

Pankoke, Eckhart, und Christoph Sachße. 1992. Armutsdiskurs und Wohlfahrtsforschung. Zum deutschen Weg in die industrielle Moderne. In Armut im modernen Wohlfahrtsstaat Kölner Zeitschrift für Soziologie und Sozialpsychologie. Sonderheft 32., Hrsg. Stephan Leibfried, Wolfgang Voges, 149-173. Opladen: Westdeutscher Verlag.

Papier, Hans-Jürgen. NZZ-online am 20.10.2020. Interview: Alexander Kissler. https://www.nzz.ch/ international/hans-juergen-papier-warnt-vor-aushoehlung-der-grundrechte-ld.1582544. Zugegriffen: 20. Okt. 2020.

Prantl, Heribert. 2008. Die Abgründe der Prävention. Zu den Mechanismen des Überwachungsstaates. Blätter für deutsche und internationale Politik 9/2008:57-67.

Raschke, Joachim. 2002. Politische Strategie. Überlegungen zu einem politischen und politologischen Kontept. In Jenseits des Regierungsalltags. Strategiefähigkeit politischer Parteien, Hrsg. Frank Nullmeier, Thomas Saretzki, 207-241. Frankfurt: Campus.

Reckwitz, Andreas. 2017. Die Gesellschaft der Singularitäten. Zum Strukturwandel der Moderne. Berlin: Suhrkamp.

Schelsky, Helmut. 1976. Die neuen Formen der Herrschaft: Belehrung, Betreuung, Beplanung. In Recht und Macht in Politik und Wirtschaft, 93-108. Zürich: Polygraphischer Verlag.

Schmidt, Manfred G. 1988. Sozialpolitik. Historische Entwicklung und internationaler Vergleich. Opladen: Leske + Budrich.

Strasser, Hermann, und Henning van den Brink. 2005. Auf dem Weg in die Präventionsgesellschaft? Aus Politik und Zeitgeschichte. https://doi.org/10.1007/978-3-531-90962-2_8.

Trampusch, Christine. 2009. Der erschöpfte Sozialstaat. Transformation eines Politikfeldes. Schriften aus dem Max-Planck-Institut für Gesellschaftsforschung Köln, Bd. 66. Frankfurt/New York: Campus.

Wehner, Christoph Julian. 2012. Grenzen der Versicherbarkeit - Grenzen der Risikogesellschaft, Atomgefahr, Sicherheitsproduktion und Versicherungsexpertise in der Bundesrepublik und den USA. Archiv für Sozialgeschichte 52:581-605.

Weingart, Peter. 1979. Das „Harrisburg-Syndrom“ oder die De-Professionalisierung der Experten. In Kernenergie: Gefahr oder Notwendigkeit. Anatomie eines Konflikts, Hrsg. Helga Nowotny, 9-17. Frankfurt/Main: Suhrkamp. 